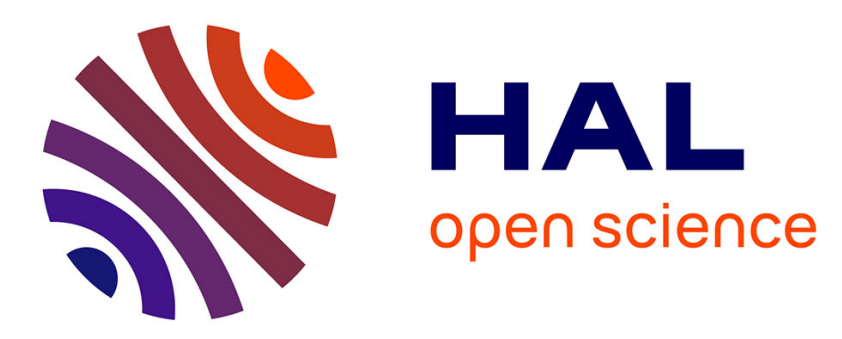

\title{
Sub-boundaries induced by dislocational creep in uranium dioxide analyzed by advanced diffraction and channeling electron microscopy
}

M. Ben Saada, Nathalie Gey, B. Beausir, X. Iltis, H Mansour, N Maloufi

\section{- To cite this version:}

M. Ben Saada, Nathalie Gey, B. Beausir, X. Iltis, H Mansour, et al.. Sub-boundaries induced by dislocational creep in uranium dioxide analyzed by advanced diffraction and channeling electron microscopy. Materials Characterization, 2017, 133, pp.112-121. 10.1016/j.matchar.2017.09.023 . hal03044975

\section{HAL Id: hal-03044975 \\ https://hal.univ-lorraine.fr/hal-03044975}

Submitted on 22 Feb 2021

HAL is a multi-disciplinary open access archive for the deposit and dissemination of scientific research documents, whether they are published or not. The documents may come from teaching and research institutions in France or abroad, or from public or private research centers.
L'archive ouverte pluridisciplinaire HAL, est destinée au dépôt et à la diffusion de documents scientifiques de niveau recherche, publiés ou non, émanant des établissements d'enseignement et de recherche français ou étrangers, des laboratoires publics ou privés. 


\title{
Sub-boundaries induced by dislocational creep in uranium dioxide analyzed by advanced diffraction and channeling electron microscopy
}

\author{
M. Ben Saada ${ }^{a, b}$, N. Gey ${ }^{a, c, *}$, B. Beausir ${ }^{a, c}$, X. Iltis ${ }^{b, * *}$, H. Mansour ${ }^{a}$, N. Maloufi ${ }^{a, c}$

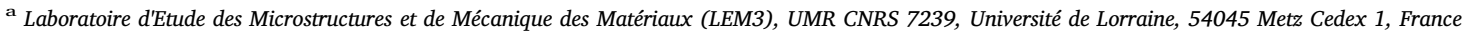 \\ b CEA, DEN, DEC, Cadarache, 13108, Saint-Paul-Lez-Durance, France \\ ${ }^{c}$ Laboratory of Excellence on Design of Alloy Metals for low-mAss Structures (DAMAS), Université de Lorraine, France
}

\section{A R T I C L E I N F O}

\section{Keywords:}

Uranium dioxide

Creep

EBSD

ECCI

Dislocation

Porous material

\begin{abstract}
A B S T R A C T
The network of sub-boundaries formed in a sintered $\mathrm{UO}_{2}$ pellet after dislocational creep was examined. Very low angle boundaries, down to $0.1^{\circ}$ were reliably detected by Electron BackScattered Diffraction (EBSD). This angular resolution was achieved by optimizing EBSD data collection and processing. Moreover, Accurate-Electron Channeling Contrast Imaging (Accurate-ECCI) was able to image the dislocations produced by creep, directly on the bulk sample. The dislocations were mostly organized in sub-boundaries with low energy configurations. Only a limited number of isolated dislocations were observed. Finally, the deformation substructure obtained after $8 \%$ creep deformation (at $1500{ }^{\circ} \mathrm{C}$ under a $50 \mathrm{MPa}$ uniaxial state) was quantified. The original grains with a mean size of $20 \mu \mathrm{m}$ were in average fragmented in sub-grains of about $5 \mu \mathrm{m}$. The geometrically necessary dislocations density (GND) was evaluated from the filtered EBSD data to $7.9 \times 10^{12} \mathrm{~m}^{-2}$. This value was 10 times higher than that measured on the as-sintered sample. This confirms that the GND density calculation is sensitive to the dislocation increase after $8 \%$ deformation by creep.
\end{abstract}

\section{Introduction}

Sintered uranium dioxide pellets are commonly used in nuclear pressurized water reactors. In normal operating conditions, their central temperature reaches values of the order of $1000{ }^{\circ} \mathrm{C}$ whereas it increases up to $1500{ }^{\circ} \mathrm{C}$, or even more, during power transients. Under these conditions, the pellets undergo significant plastic deformation through creep mechanisms, which influence pellet-cladding interaction processes $[1,2]$. These mechanisms are partly reproduced by compression creep tests on non-irradiated pellets [3]. The resulting creep damage in these deformed $\mathrm{UO}_{2}$ pellets is characterized by the development of inter-granular cavities at low stresses (25 MPa), and crack opening at high stresses (50 MPa) [3]. Inside the grains, dislocations produced during the deformation arrange in sub-boundaries, as previously evidenced by means of Transmission Electron Microscopy (TEM) examinations [4]. However, no scale transition between the SEM observations on bulk $\mathrm{UO}_{2}$ crept samples and those made by TEM on thin foils, can be found in the literature. In addition, no quantified data about the deformation substructure is available.

Electron BackScattered Diffraction (EBSD) and Electron Channeling Contrast Imaging (ECCI) could contribute to achieve TEM-SEM scale transitions and quantify the microstructure evolution under creep. Indeed, ECCI is a SEM-based technique that allows imaging defects in the near surface of bulk materials [5-8]. Moreover, in many cases, comprehensive characterization of dislocations is possible using analyses of dislocation contrast with various diffraction conditions [9-17]. Nevertheless, acquiring high quality ECC images requires the channeling conditions to be precisely satisfied. Electron Channeling Pattern (ECP) limited to single crystals or Selected Area Channeling Patterns (SACP) more suitable for polycrystalline materials are used to control the orientation of the region of interest. Unfortunately, the poor spatial resolution of SACP restricts ECCI applications to large grained materials $[9,11,18-25]$. A new approach developed recently allows the collection of High Resolution SACPs with a spatial resolution of $500 \mathrm{~nm}$ [26]. It opens the way to apply Accurate-ECCI on submicron sized grains [27]. Dislocations in creep deformed $\mathrm{UO}_{2}$ can now be observed by A-ECCI as demonstrated in [28] while in the past they were only analyzed by TEM $[3,4]$. Furthermore, continuous progress in EBSD detectors and diffraction patterns analysis offer now improved angular resolution to reliably quantify the low angle boundaries and the subsequent network of geometrically necessary dislocations (GND) [29-33].

Thus, this paper performs up to date EBSD analysis and A-ECC

\footnotetext{
* Correspondence to: N. Gey, Laboratoire d'Etude des Microstructures et de Mécanique des Matériaux (LEM3), UMR CNRS 7239, Université de Lorraine, 54045 Metz Cedex 1, France.

** Corresponding author.

E-mail addresses: nathalie.gey@univ-lorraine.fr (N. Gey), xaviere.iltis@cea.fr (X. Iltis).
} 
Imaging on $\mathrm{UO}_{2}$ samples to analyze and quantify the deformation substructure induced by dislocational creep. EBSD data were acquired in improved angular resolution mode and post-processed with a routine optimized for porous materials. As a result, very low angle boundaries disorientated down to $0.1^{\circ}$ were detected. These sub-boundaries were further observed by A-ECCI to reveal the dislocation network induced by creep deformation. Finally EBSD-based quantities were calculated over large EBSD maps to quantify the deformation substructure at a given creep state. They include the quantification of linear fraction of sub-boundaries and boundaries depending on their disorientation range, the determination of sub-grain average size and the GND density quantification. These quantities are worth to quantify the sub-structured microstructure induced by creep and compare its evolution for different deformation conditions. They may also be useful in physicallybased modeling of the creep behavior.

\section{Experimental}

\subsection{Material}

Pellets with a height of $12 \mathrm{~mm}$ and a diameter of $8 \mathrm{~mm}$ were fabricated from a single batch of $\mathrm{UO}_{2}$ powder [34]. The powder was pressed uniaxially under $400 \mathrm{MPa}$ and sintered at $1900{ }^{\circ} \mathrm{C}$ for $4 \mathrm{~h}$ under $\mathrm{Ar}+5 \% \mathrm{H}_{2}$ atmosphere (thereby ensuring the material stoichiometry). Their density measured by an immersion method was $98.6 \%$ of the theoretical density of $\mathrm{UO}_{2}$.

Fig. 1(a) illustrates the microstructure of the as-sintered reference sample. The grains are revealed by orientation contrast in backscattered electron (BSE) imaging mode. The average grain size is of $20 \mu \mathrm{m}$. Small round fabrication voids are also present: they are located in inter- and intra-granular positions. Intra-granular pores are smaller (mean size of $0.4 \mu \mathrm{m}$ ) and more numerous than inter-granular ones.

A compression creep test was conducted in an adapted furnace on a screw-type Instron compression test machine. The pellet was crept in the dislocational regime at $1500{ }^{\circ} \mathrm{C}$ under $50 \mathrm{MPa}$ [34]. The steady creep state was reached after 80 min with a mean strain rate of $2.7 \% / \mathrm{h}$. The experiment was interrupted after $3 \mathrm{~h}$ at a mean deformation of about $8 \%$. The same atmosphere as for sintering was applied to maintain the material stoichiometry.

Finally, the pellet was sectioned parallel to the compression axis and the most deformed area in the central part of the pellet was identified by SEM examinations. Fig. 1b illustrates the microstructure after creep deformation. Important variations in the BSE signal are observed inside the grains and evidence sub-grains induced by the plastic deformation. Creep damage like large intergranular cavities is also observed.

\subsection{Optimized EBSD Data Acquisition and Processing}

EBSD maps were acquired using a FEI NovaNano SEM 450, equipped with a Nordlys II Nano camera (maximum CCD resolution: $1344 \times 1024$ pixels) and the process-driven software AZTEC (from Oxford Instruments). The EBSD data were acquired at an acceleration voltage of $20 \mathrm{kV}$, a beam current of about $10 \mathrm{nA}$, and a working distance of $15 \mathrm{~mm}$. The diffraction patterns were indexed with a $\mathrm{CaF}_{2}$ type fluorite structure (space group $225-F m \overline{3} m$ ). Indeed, uranium dioxide crystals form an FCC uranium lattice ( $\mathrm{a}=5.4705 \AA$ ) with oxygen ions located in the tetrahedral interstitial sites.

To improve the angular resolution, EBSD maps were performed at low camera binning of $2 \times 2$ and the Kikuchi patterns were indexed with the 'refined accuracy' mode in AZTEC software [35]. This mode improves the Kikuchi Band detection: it considers that the Kikuchi bands are hyperbolic and refines the fit between experimentally detected and simulated bands accordingly. The angular resolution of conventional EBSD data is improved without severe impact on the acquisition speed.

Two types of maps were acquired. The first type corresponds to

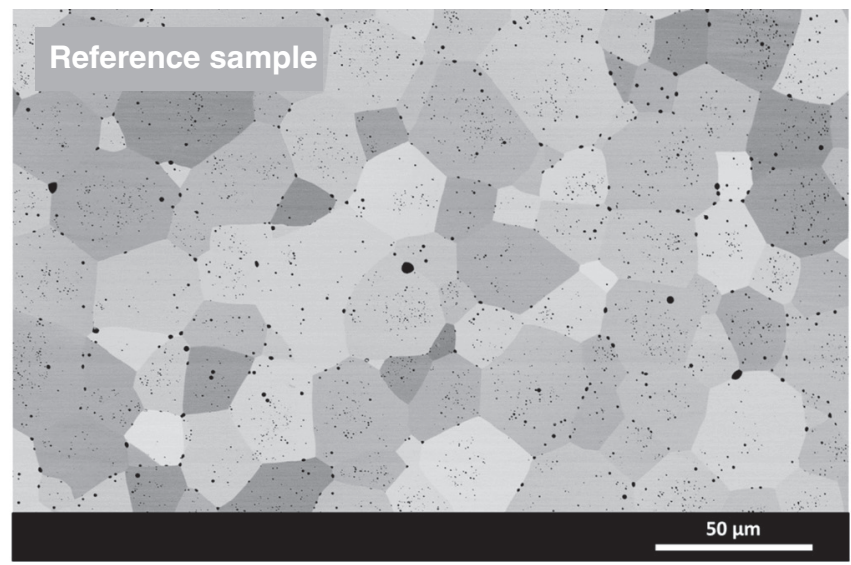

(a)

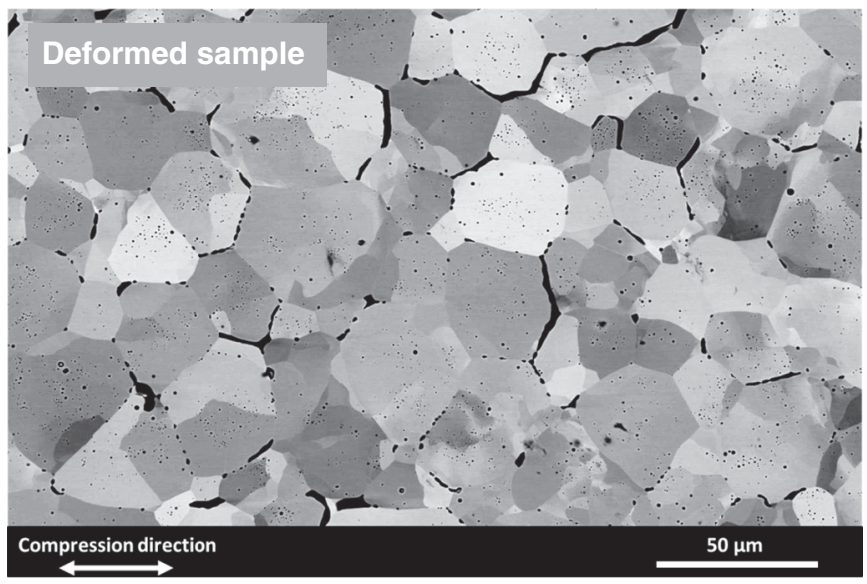

(b)

Fig. 1. BackScattered Electron micrographs of $\mathrm{UO}_{2}$ (a) "as-sintered" sample and (b) deformed sample.

small-scale maps with a reduced step of $0.15 \mu \mathrm{m}$ to reveal the network of intragranular misorientation induced by creep. The second type corresponds to large scale maps performed with an acquisition step of $0.4 \mu \mathrm{m}$. These maps cover each around 300 grains for statistical analysis of the orientation data. Only one large EBSD map was acquired on the reference sample (named R0) whereas three maps (named D1, D2 and D3) were acquired on the deformed sample. They were measured in the central part of the sample where the most damaged area was located. All these EBSD maps had an indexation rate > 98\%.

We paid a particular attention to the voids; they were all partially indexed whereas they should not. Fig. 2 shows an example of an EBSD map acquired over a void previously imaged at $70^{\circ}$ tilt (Fig. 2a). Most of the void surface was indexed as seen in Fig. 2b. Moreover the orientations obtained within the void were disoriented by $3^{\circ}$ (in yellow/ green) from the orientation of its surrounding (in blue). These disorientations are artificial; they are caused by the topography around and within a void. Consequently, the orientations measured over the voids must be removed from the data set for a reliable analysis of disorientation induced by the creep process.

Thus a specific procedure was developed to clean EBSD data acquired on porous material. Its objective was to assign the status 'nonindexed' to points measured over voids. The most efficient EBSD-based index to detect the void location on the EBSD map was found to be the Band Slope (BS). The BS is a measure of the Kikuchi band sharpness. It is based on the diffraction intensity profile between the band edges: the sharper the intensity variation, the higher the BS value. Its average value over the voids was significantly different from that over the $\mathrm{UO}_{2}$ 


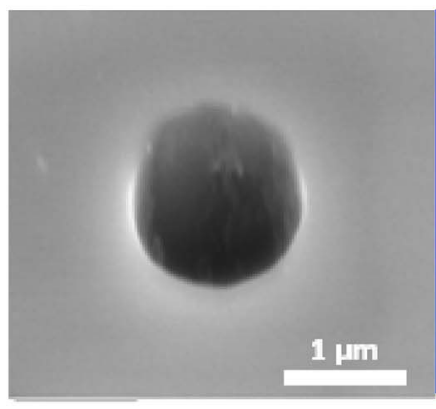

(a)

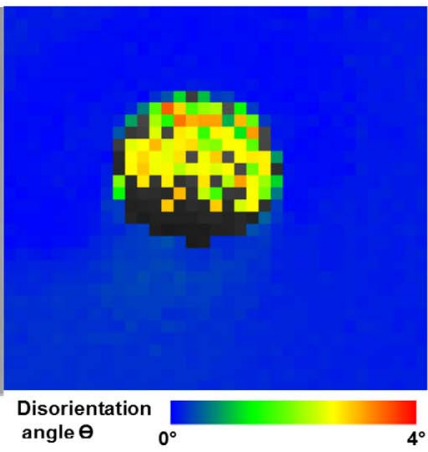

(b)

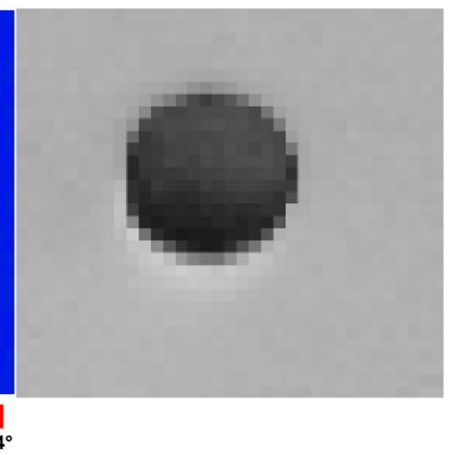

(c)
Fig. 2. Example of EBSD map over a void (a) SEM image at $70^{\circ}$ tilt, (b) Corresponding orientation map (the color code indicates the deviation from the average orientation of the blue grain) and (c) band slope map. (For interpretation of the references to color in this figure legend, the reader is referred to the web version of this article.) matrix, as seen in Fig. 2c. Thus it could easily be thresholded to locate the voids. For our operating conditions, the threshold value was set to 150 (BS values range from 0 to 255). Pixels with BS below 150 were assigned to the status 'nonindexed'. They were mostly associated to voids, but also to some pixels located at prior $\mathrm{UO}_{2}$ boundaries.

Additional 'dilate' and 'erode' data processing steps ${ }^{1}$ were applied to reconstruct locally some boundaries wrongly set to 'nonindexed' without final changes of the void's contours.

Finally the angular resolution of the EBSD data was further improved by applying a local averaging filter. It was developed in Atom software [36] by adapting the Kuwahara filter (well known in image processing) to orientation data $[37,38]$. One moves a kernel of $5 \times 5$ pixels over the EBSD map. This kernel is then divided into four regions of $3 \times 3$ pixels (one from each corner of the $5 \times 5$ kernel) and the disorientation between the indexed pixels are calculated. The central pixel of the kernel is then reoriented to the average orientation of the region displaying the smallest standard deviation in disorientation distribution. This filter efficiently reduces the orientation noise while preserving continuous disorientation lines.

\subsection{Accurate ECCI to Reveal Dislocation Sub-boundaries}

We used A-ECCI to reveal the dislocations induced by creep and their arrangement in sub-boundaries [27,39]. High-resolution selected area channeling patterns (HR-SACPs) were collected in the Zeiss Auriga 40 FEG-SEM equipped with the GEMINI-type electron column. All images were acquired at a working distance of $7 \mathrm{~mm}$, an acceleration voltage of $20 \mathrm{kV}$ and a beam current of $550 \mathrm{pA}$ (with the $30 \mu \mathrm{m}$ center aperture). The rocking beam mode was calibrated to obtained submicron resolution SACPs as demonstrated in [26]. This resolution allows acquiring HR-SACPs over the $\mathrm{UO}_{2}$ sub-grains.

Fig. 3a gives an example of HR-SACP acquired over the $\mathrm{UO}_{2}$ sample at zero tilt. The HR-SACP is indexed manually using the Kikuchi pattern simulated from the approximate orientation deduced by EBSD (Fig. 3b, c). To reach the desired two-beam channeling conditions and image the dislocations in the grain of interest, the sample is then rotated and tilted using the precise information extracted from the HR-SACP. Finally at the expected channeling position, a last HR-SACP is acquired to confirm the sample orientation relative to the incident beam before ECCI analysis.

As seen Fig. 3a, the intensity distribution of an experimental SACP of $\mathrm{UO}_{2}$ is extremely complex and the SACP can be indexed only by

\footnotetext{
1 'Erode' and 'dilate' are morphological transformations classically applied to binary images.

They are often used to clean EBSD data. Here, the 'dilate' transformation is first applied to extend indexed regions over neighboring non-indexed regions (the pores and the nonindexed grain boundaries). Then an addition 'erode' transformation is applied to extend the non-indexed regions over their indexed neighborhoods. This puts the largest pores back to their original size without any impact on the fully reconstructed grain boundaries.
}

comparison with a dynamical simulation. In this contribution, these simulations were obtained with "Esprit DynamicS" software from Bruker (the default setting for $\mathrm{UO}_{2}$ phase was used for the input parameters) [40]. Fig. 3b and c show respectively the dynamic and kinematic patterns simulated from the approximate orientation deduced by EBSD. While it is difficult to locate unambiguously the HR-SACP on the kinematic simulation, the dynamic one offers a reliable and precise location of the HR-SACP. Of course further optimization of the software input parameters (absorption, excitation lengths) would be required to reproduce exactly the intensity distribution of the experimental SACP.

\section{Results and Discussion}

\subsection{Creep Induced Sub-boundaries Detected on Improved Angular Resolution EBSD Map}

Increasing the creep deformation produced a pronounced network of sub-boundaries inside the prior $\mathrm{UO}_{2}$ grains. This is easily evidenced on the BSE micrograph of the $\mathrm{UO}_{2}$ deformed sample, by the contrast variations observed within each prior grain (Figs. 1b, 4a). Detection of this network by EBSD requires optimizing data acquisition and processing to get the best angular resolution of the EBSD map. Our protocol detailed in Section 2.2 is able to resolve very low angle boundaries disoriented down to $0.1^{\circ}$. Fig. $4 \mathrm{~b}$ shows the disorientation map obtained over the region imaged in Fig. 4a (step size: $0.15 \mu \mathrm{m}$ ). The network of low and very low angle boundaries measured within the prior grains overlaps quite well the sub-boundary network visible on the BSE image (Fig. 4a). Most of the disorientation lines of $0.1^{\circ}$ are confirmed to be reliable sub-boundaries, actually observed in the BSE image.

The comparison of Fig. $4 \mathrm{~b}$ and $\mathrm{c}$ illustrates the benefits of the proposed data processing. On the raw EBSD map (Fig. 4c), most of the pores visible on the micrograph (Fig. 4a) have been indexed, leading to artificial disorientations in their neighborhood. On the other hand, on the BS-threshold map (Fig. 4b), most of the voids are identified and not indexed. Moreover, on the raw EBSD map, the sub-boundaries are embedded in the orientation noise whereas on the filtered map, they are resolved.

Traditionally, Hough-transform based EBSD map offers an angular resolution of about $0.5^{\circ}$ to $1.5^{\circ}$ depending on the material and the SEM/ EBSD settings [40]. High angular Resolution EBSD (HR-EBSD) was developed to measure relative strain and rotation with an improved angular resolution [41-44]. It uses cross correlation based techniques to measure very small EBSD pattern shifts and provides theoretical precision of about $10^{-4} \mathrm{rad}\left(0.006^{\circ}\right)$. In practice, disorientations down to $0.1^{\circ}$ were detected in a Ni-based superalloy [43]. The ultimate precision is reached only with high quality EBSD patterns acquired with a long exposure time (often $1 \mathrm{~s}$ per point). It is thus developped to estimate the strain tensor with a high accuracy but not to index large EBSD datasets (due to the drawback of contamination and drift for large area maps). 


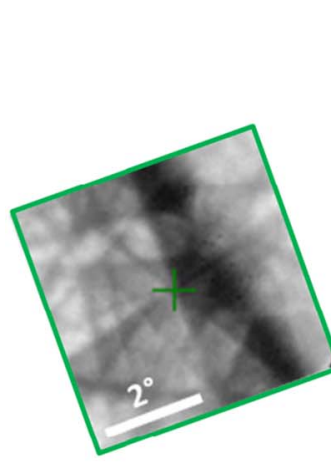

(a)

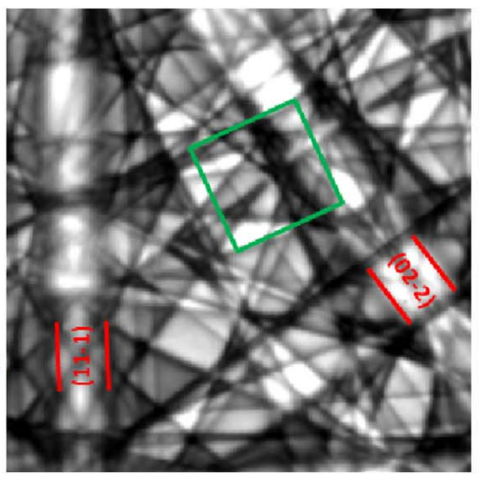

(b)

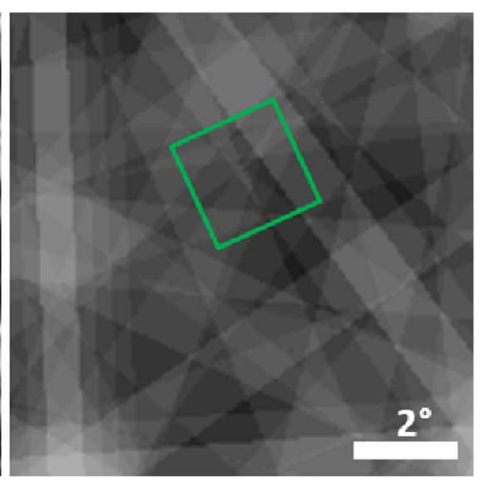

(c)
Fig. 3. (a) HR-SACP collected from $\mathrm{UO}_{2}$, (b) the corresponding pattern simulated dynamically and (c) kinematically. The green cross in the HRSACP indicates the microscope optic axis. (For interpretation of the references to color in this figure legend, the reader is referred to the web version of this article.)
The present indexing strategy is more inspired by the '3D Hough transform' approach developed by Maurice and Fortunier and for which a theoretical resolution of $5.10^{-5} \mathrm{rad}\left(0.003^{\circ}\right)$ is expected [41]. On $\mathrm{UO}_{2}$ samples, with an acquisition speed of 20 points/s (20 times higher than the conventional one used for HR-EBSD), an angular resolution of $0.1^{\circ}$ was obtained. So, the proposed approach provides a real alternative to quantify precisely low angle sub-boundaries, down to $0.1^{\circ}$, with an interesting compromise between step size and exposure time.

\subsection{Creep Induced Dislocation Network Observed by A-ECCI}

Different sub-grained regions, black boxed in Fig. 4b, were further analyzed by A-ECCI. Several channeling conditions have been applied to image dislocations configurations consisting of several Burgers vectors. Figs. 5 to 7 give typical images of these sub-boundaries for one single channeling condition. They visualize different arrangements of dislocations induced by creep deformation in low and very low angle boundaries as well as isolated dislocations.

First, these ECC-images clearly confirm that the very low angle boundaries detected by EBSD trace the pile-up of dislocations. Fig. 5a gives a typical example of ECC-image acquired over a region containing a $0.1^{\circ}$ disoriented line measured by EBSD (Fig. 5b). The low-disorientation lines in the EBSD map perfectly overlap the dislocation arrangement revealed by ECCI. Such dislocations are usually referenced as "Geometrically Necessary Dislocations" (GND): they induce a lattice curvature in order to accommodate local deformation gradients induced by creep and rearrange in low angle boundaries by further annealing.

According to our experience, for $\mathrm{UO}_{2}$ grain boundary dislocations can be distinguished individually by ECCI for sub-boundaries disoriented up to $0.8^{\circ}$, for $\mathrm{UO}_{2}$ samples. Fig. 6 illustrates by ECCI the transition between sub-boundaries with regularly spaced dislocations countable or not respectively. The different sub-grains imaged in Fig. 6a have either disorientations (Fig. 6b) belonging to very low $\left(<1^{\circ}\right.$, in yellow) or low angle boundaries ( $>1^{\circ}$ and $<5^{\circ}$, in green).

The disorientation of a boundary is related to the spacing between Geometrically Necessary Dislocations, the higher the disorientation the smaller the spacing. For a model boundary made up of edge dislocations (tilt boundary), the low angle boundary disorientation $(\theta)$ and the dislocation's spacing (D) are related by [45]:

$\theta=\frac{|b|}{D}$

where $\mathrm{b}$ is the Burgers vector $(|\mathrm{b}|=\mathrm{a} / 2\langle 110\rangle=0.387 \mathrm{~nm}$ for $\mathrm{UO}_{2}$ ).

The yellow line between sub-grains 1 and 2 has a disorientation angle of $0.36^{\circ} \pm 0.05^{\circ}$. On the corresponding ECC image (Fig. 6a), the average dislocation's spacing is $57 \pm 5 \mathrm{~nm}$. Those values are consistent with that expected for a theoretical tilt boundary. However, further analysis is of course required for a complete description of this subboundary. On the contrary, the green sub-boundary between sub-grains 2 and 3 disoriented by $1.6^{\circ}$ has no individual dislocations visible (a spacing of $14 \mathrm{~nm}$ would be required for a tilt boundary model) (Fig. 6b).

Further ECC-images indicate that most of the dislocations induced by the deformation were organized in sub-boundaries; only a limited number of isolated dislocations were observed within the sub-grains (some are seen in Fig. 6). Moreover, they were mostly organized in low energy configurations. Fig. $7 \mathrm{a}$ and $\mathrm{b}$ show a faceted sub-boundary (disoriented from $0.33^{\circ}$ to $0.74^{\circ}$ ). Fig. $7 \mathrm{c}$ shows a sub-boundary made of a regular network of dislocations (disoriented by $0.1^{\circ}$ ). Such networks are typical of boundaries incorporating different sets of screw dislocations. If the Burgers vectors are orthogonal then the dislocations do not strongly interact and the dislocation lines form a square network in the grain boundary plane. If the two Burgers vector can interact and form a third small Burgers vector then the dislocations produce a honeycombed network [46]. These different configurations have already been observed in crept $\mathrm{UO}_{2}$ by TEM $[3,4]$. The honeycombed network was interpreted as initial $\langle 110\rangle$ screw dislocations gliding in $\{001\}$ planes $^{2}$ and interacting to form a third family by cross slip in the $\{111\}$ plane. More generally such organized grain boundary structures are assumed to be obtained with dislocation climbing out of their glide plane [46]. This mechanism is consistent with the high creep temperature $\left(1500^{\circ} \mathrm{C}\right)$ at which fast diffusion is operating.

\section{EBSD-based Parameters to Quantify the Substructure Induced by Creep}

In this section, we introduce different EBSD-based parameters to analyze quantitatively the grain substructure that develops during creep. These parameters are calculated on large scale EBSD maps, to be statistically relevant (see Section 2.2). They are worth for coming research, to compare the microstructure evolution for different creep deformation conditions. They may also be useful in the development of physically-based modeling of the creep behavior [49].

\subsection{Sub-boundary Quantification at Large Scales}

A dense network of sub-boundaries was created during creep. Most of them were disoriented by $<1^{\circ}$. However some limited sub-boundaries reached a disorientation up to $5^{\circ}$. This is seen on Fig. $8 \mathrm{a}$ and b with the disorientation-maps obtained on the reference sample and on the

\footnotetext{
${ }^{2}$ Dislocations in $\mathrm{UO}_{2}$ are characterized by the Burgers vector $\mathrm{b}=\mathrm{a} / 2\langle 110\rangle$. Due to the electrostatic interactions of ions within dislocation cores, the glide systems are not those classically observed in FCC structure. Indeed, $\{100\}\langle 110\rangle$ is the easiest system in $\mathrm{UO}_{2}$. With increasing temperature and deformation, $\{110\}$ glide planes are also activated, as well as $\{111\}$ planes by cross slip $[47,48]$.
} 
(a)

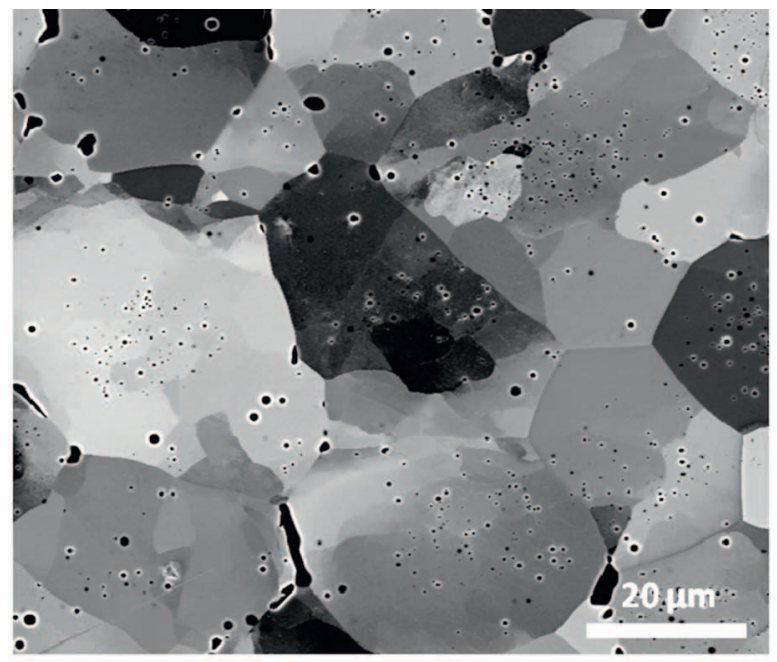

(b)

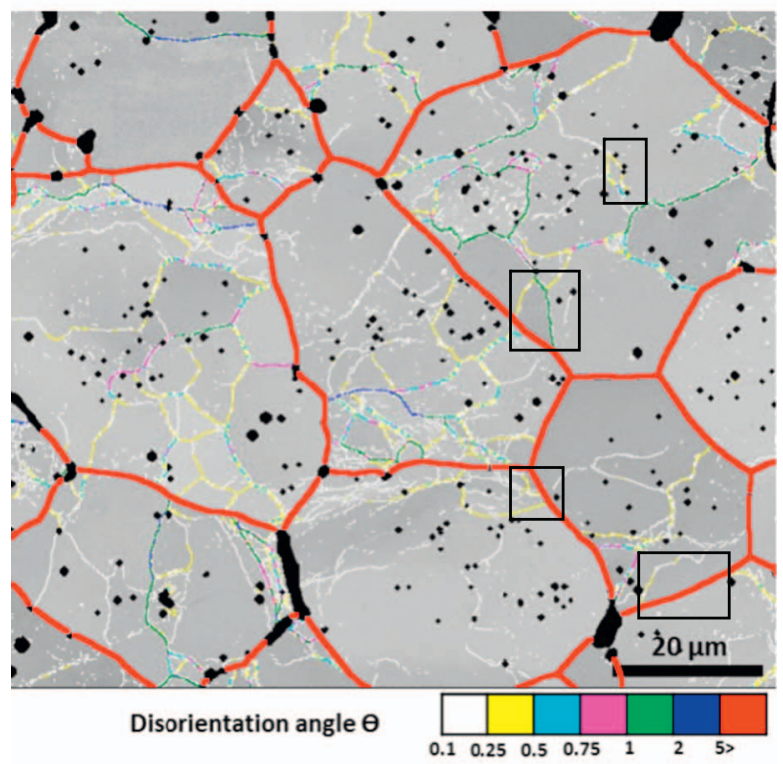

(c)

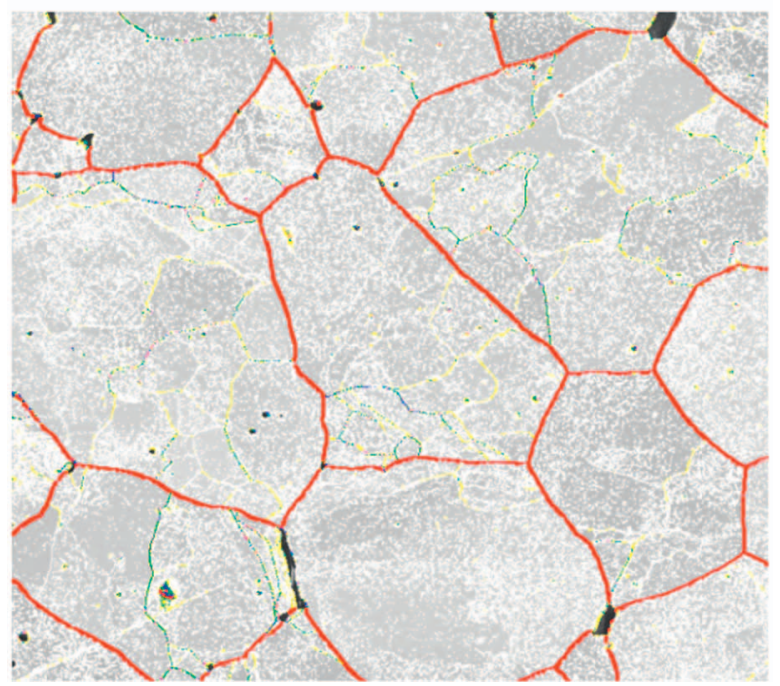

Fig. 4. Deformed sample: (a) BSE micrograph, Disorientation angle map of the (b) filtered and (c) raw data (the background gray level is given by the Kikuchi Pattern Quality index, non-indexed points are black). crept sample (filtered data). The same color code as in Fig. 3 is applied, except that the smallest disorientation class, with angles below $0.25^{\circ}$, is not considered.

This network was further quantified by calculating the linear fractions of boundaries and sub-boundaries associated to different disorientation classes. Fig. 9 considers all disorientations $>0.25^{\circ}$ and classifies them in four groups. In the reference sample (R0 map), most of these disorientations were higher than $15^{\circ}(96 \%)$. They characterize the disorientations between the $\mathrm{UO}_{2}$ grains prior to deformation. Only $1.3 \%$ of the considered disorientations were lower than $5^{\circ}$. Conversely, in the $\mathrm{UO}_{2}$ deformed sample (maps D1, D2 and D3), the fraction of subboundaries with disorientations lower than $5^{\circ}$ reached 68\% (among which $52 \%$ are disoriented by $<1^{\circ}$ ). This increase from 1.3 to $68 \%$ quantifies the network of sub-boundaries induced by the deformation. It is interesting to notice that the three areas mapped by EBSD in this sample lead to very similar results. This allows concluding that each EBSD map is large enough to characterize the substructure induced by deformation. Of course, this holds only for EBSD maps acquired in the central part of the pellet, knowing that the deformation is not homogeneous at the whole pellet scale.

On the large EBSD maps, the disorientation class, with angles below $0.25^{\circ}$ were not quantified because these disorientations were not only associated to sub-boundaries induced by creep, but also to disorientation lines, induced by scratches coming from mechanical polishing of the sample. In addition, on these EBSD maps acquired with a larger step size $(0.4 \mu \mathrm{m})$, the pores are less resolved and consequently the disorientation noise around them cannot be completely eliminated.

The disorientations belonging to the noise were further quantified and the result confirms that local disorientations below $0.25^{\circ}$ were mostly embedded in the noise on the large-scale maps. This result was obtained by processing the EBSD data of the reference sample as follows. The orientation spread was calculated within the largest grains (with equivalent diameter $>5 \mu \mathrm{m}$ ). For each detected grain, one calculates from all orientations measured within this grain: the grain average orientation, the average grain orientation spread A-GOS (average disorientation from all measurements to the average orientation), the maximum grain orientation spread M-GOS (maximum disorientation from all measurements to the average orientation). Table 1 gives the statistic obtained over 200 recrystallized grains for the filtered and unfiltered data. Considering the upper bond of the $3 \sigma$ confidence interval, one obtains respectively for the unfiltered/filtered data an AGOS value of $0.1^{\circ} / 0.05^{\circ}$ and a maximum GOS value of $0.32^{\circ} / 0.25^{\circ}$. This indicates that the orientation noise is in average equal to $0.1^{\circ} / 0.05^{\circ}$ on the large EBSD maps but locally it can reach up to $0.32^{\circ} / 0.25^{\circ}$ (unfiltered/filtered data).

\subsection{Quantification of the Sub-grain Sizes}

The polygonization of the $\mathrm{UO}_{2}$ deformed grains can be further quantified by identifying the sub-grains on the EBSD map and evaluating their average size. The classical EBSD grain recognition procedure clusters pixels disoriented from their neighbor by less than a threshold angle $\omega_{0}$. However, a reliable detection of continuous sub-boundaries with disorientations close to the angular resolution of the map requires specific attention. Indeed, the classical procedure would require to set $\omega_{0}$ close to the orientation noise but would identify noisy pixels as subgrains. Conversely, increasing $\omega_{0}$ value would produce artificial open sub-boundary lines and consequently two sub-grains would be clustered in a single one. To overcome this problem, an optimized grain detection procedure has been proposed for sub-boundary identification [50,51]. It proceeds in two steps and defines an upper and lower bound for the threshold angle $\omega$. The upper value is set higher than the orientation noise and is used to identify a first network of well-identified boundary and sub-boundary lines. However those lines can be discontinuous, due to the 'high' value of $\omega$. The second step aims to close the open lines by tracking a path between neighboring pixels considering disorientation 


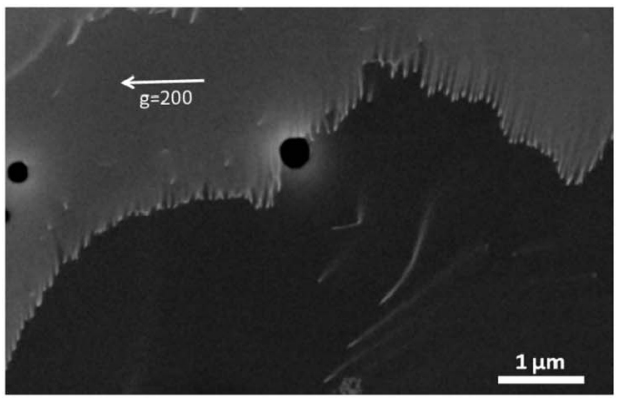

(a)

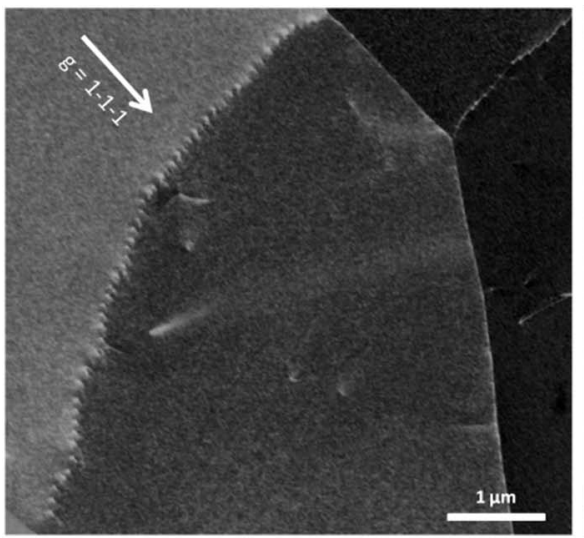

(a)

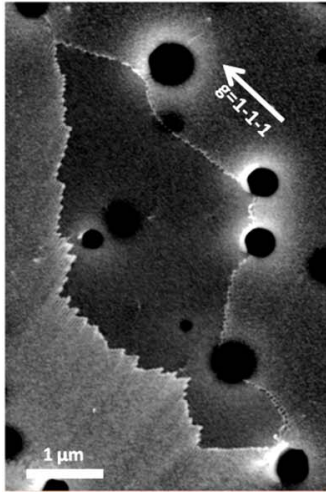

(a)

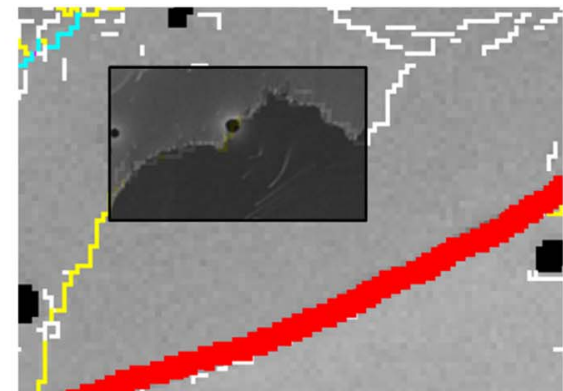

Disorientation angle $\theta$ (b)

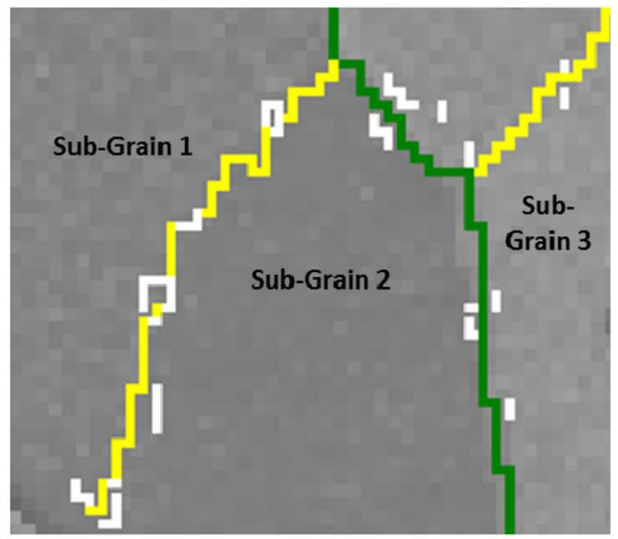

Fig. 6. (a) A-ECC image of a very low (left side) and a low angle boundary (right side) with respectively countable and uncountable dislocations and (b) corresponding EBSD map (zoom from Fig. 4), the sub-boundaries are presented with similar color code as in Fig. 4. (For interpretation of the references to color in this figure, the reader is referred to the web version of this article.)
Fig. 5. (a) A-ECC-image over a very low angle boundary detected on the EBSD map in Fig. 4 and zoomed in (b).

(b)

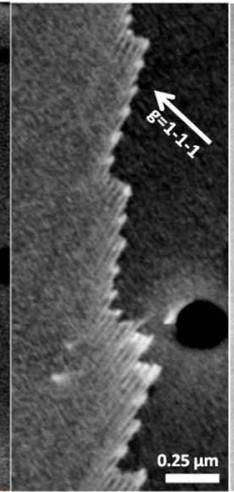

(b)

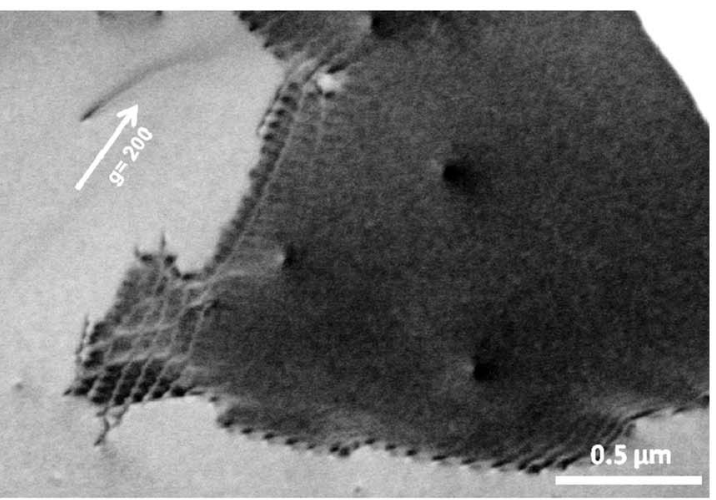

(c)
Fig. 7. A-ECC image at high resolution showing different types of dislocation arrangements (a-b) faceted sub-boundaries disoriented of $0.33^{\circ}-0.74^{\circ}$, (c) a dislocation network with disorientations down to $0.1^{\circ}$. angles down to the lower bound.

This optimized sub-grain detection procedure was applied on the crept sample with the critical angle $\omega$ set to $0.25^{\circ}$, for the upper value and $0^{\circ}$, for the lower one. The initially $20 \mu \mathrm{m}$ large grains were found to be fragmented into sub-grains with an average size of about $5 \mu \mathrm{m}$. Fig. 8c highlights in random colors sub-grains identified within the prior $\mathrm{UO}_{2}$ grains contoured in red. They rarely form a homogeneous network within the prior grains but tend to concentrate at their boundaries. This probably results from heterogeneous creep deformation with higher strain incompatibilities at those prior boundaries. Testing conditions (in particular, the mean strain rate) and the original microstructure (grain size, pores distribution...) probably also affect this sub-structuration process.

\subsection{Geometrically Necessary Dislocations Analysis}

In this section, the increase in GND density with creep deformation was quantified from the large scale EBSD maps (unfiltered/filtered data). In fact, among the dislocations induced by plastic deformation and previously observed by ECCI, one distinguish the SSD (statistically stored dislocations) et GND. A part of the density of the GND can be obtained from orientation maps [52] through the Nye tensor $\alpha$ [53]:

$\alpha=\operatorname{curl}\left(\varepsilon_{e}\right)+\operatorname{tr}(\kappa) I-^{t} \kappa$

The term $\operatorname{curl}\left(\varepsilon_{e}\right)$ will be here overlooked as the elastic deformation $\varepsilon_{e}$ cannot be extracted from orientation maps. $\kappa$ is the elastic curvature tensor, calculated from the EBDS map: 


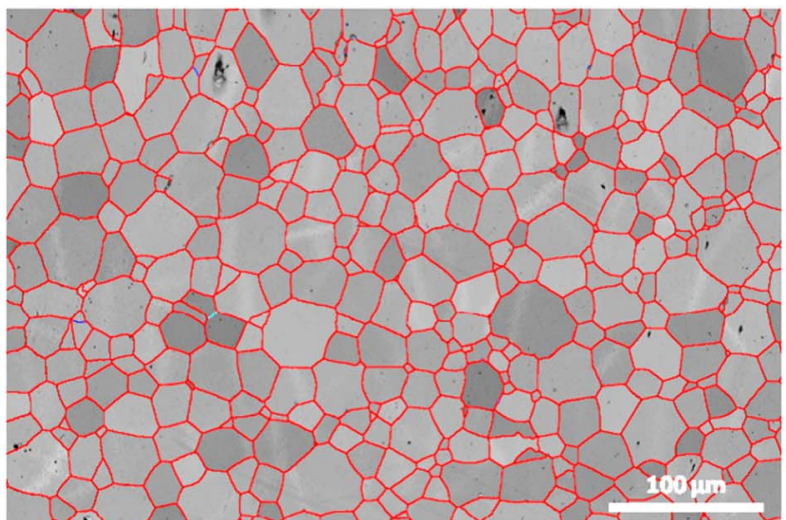

(a)

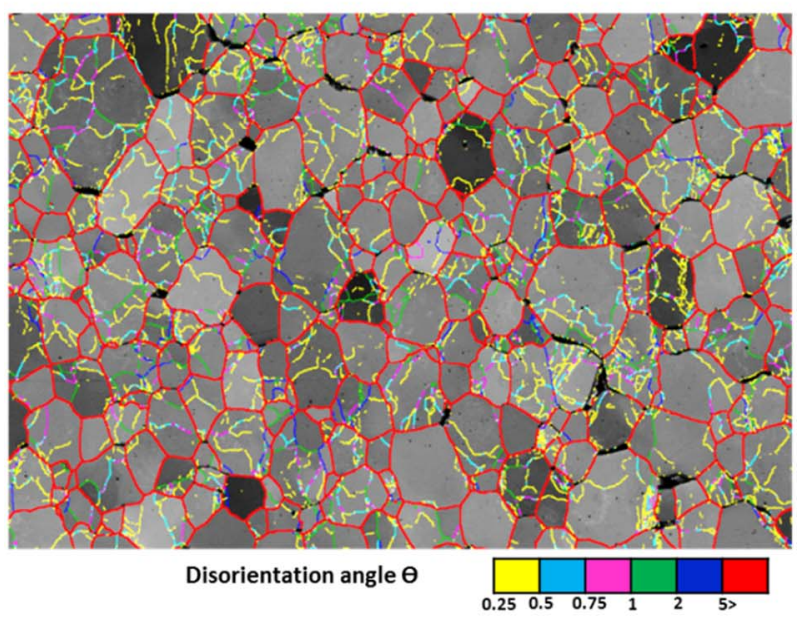

(b)

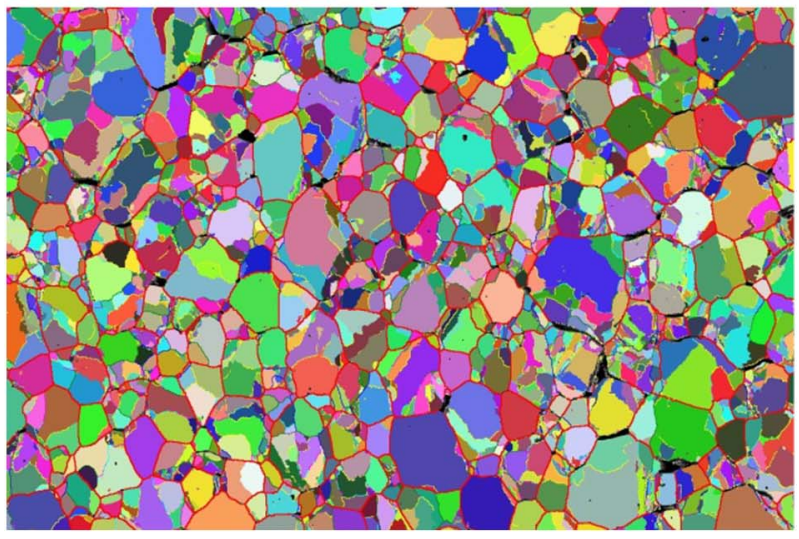

(c)

Fig. 8. Disorientation map overlapped to the Kikuchi pattern quality map (filtered data) for (a) the reference sample, (b) the deformed sample and (c) random coloring of subgrains identified with an optimized routine - deformed sample. (For interpretation of the references to color in this figure, the reader is referred to the web version of this article.)

$\kappa_{i j} \approx \frac{\Delta \theta_{i}}{\Delta x_{j}} ; \quad \forall i \in(1,2,3), \quad \forall j \in(1,2)$

$\Delta \theta_{i}$ being the components of the disorientation vector between neighboring points separated by $\Delta x_{j}$.

Different methods are then used to evaluate the dislocation density [54-58].

In this contribution, the calculation was performed with ATOM software [36]. The elastic curvature tensor (Eq. (3)) was calculated with the two point finite difference approximation (considering the current pixel with its right- and bottom-neighbor). A cut-off angle of $5^{\circ}$ was applied for translating a disorientation boundary into GND. The dislocation density is expressed by the entrywise 1-norm of the Nye tensor:

$\rho_{\mathrm{GND}}=\frac{1}{b} \sqrt{\alpha_{i j} \alpha_{i j}}$

However, from 2D maps, only six components of the elastic curvature tensor can be captured, as differences along the third direction are not available. Note also that the norm is divided by the Burger's vector length to present density values in $\mathrm{m}^{-2}$ unit.

Fig. 10 maps the GND density in terms of entrywise norm of the Nye tensor for the deformed sample (filtered D3 map). Dislocation densities higher than $12.9 \times 10^{13} \mathrm{~m}^{-2}$ are all colored in red. These highest densities (with a maximum reaching $89 \times 10^{13} \mathrm{~m}^{-2}$ ) arise from the most disoriented sub-boundaries (those disoriented by $>2^{\circ}$, colored in blue and red in Fig. 8b). Table 2 gives the average dislocation densities $\left\langle\rho_{\mathrm{GND}}\right\rangle$ calculated from the EBSD maps of the reference and the deformed sample (unfiltered and filtered data set). In average, the dislocation density of the deformed samples is of $17 \times 10^{12} \mathrm{~m}^{-2}$. Interestingly, the calculated values over the three deformed maps are very close (Table 2). This confirms again that each map is large enough to be representative of the deformed state at the central part of the pellet.

However, the absolute level of GND must be considered with caution as it changes significantly according to various parameters $[54,57,59,60]$. It is worth to mention that the GND calculation is step size dependent (see Eq. (3)). It is also sensitive to the precision of the orientations measured by EBSD. The use of the Kuwahara filter reduces the orientation noise in the EBSD data. Consequently the GND values calculated from the unfiltered and filtered EBSD data show large differences (up to a decade as seen in Table 2). The effect of different filters on the GND values has been discussed in [60]. The authors apply the calculation to orientation data obtained from as-acquired patterns and from as-acquired patterns plus an additional noise. All tested filters brought the overall calculated GND distribution closer to that calculated without additional noise. This result clearly shows that the application of a filter mitigates for averaged GND densities the effect of orientation precision. However such filter modifies locally the EBSD data by considering the local neighborhood. But for the dislocation density field, this is clearly not true. Thus for a discrete, and local GND analysis of the structure of the sub-grain boundary, it is recommended to consider the unfiltered EBSD data. However to be reliable, such local GND analysis requires orientation data with a high precision; HREBSD would be recommended.

Whatever, this orientation noise is translated in a certain level of dislocation density noise, often called spurious density [54]. As a consequence, the analysis of GND density values makes sense only if the level of spurious densities $\rho_{\text {sp }}$ is also quantified. In this study, we suggest to evaluate the spurious densities $\rho_{\mathrm{sp}}$ from the noisy disorientations obtained on a non-deformed sample (that contains no dislocations induced by the applied deformation). Of course the data must be acquired with similar acquisition parameters as for the deformed sample. The upper bound of the disorientation noise $-0.25^{\circ}$ - (Table 1 , Section 4.1) gives the range to translate a disorientation to noisy dislocation densities. This approach was applied to the large scale EBSD map of the reference sample. Disorientations below $0.25^{\circ}$ were translated in the noise level of the dislocation density.

Applied to the reference map, the average spurious density of dislocations was evaluated for our EBSD data to $11.5 \times 10^{12} \mathrm{~m}^{-2}$ (unfiltered data in Table 2). The calculated average GNDs density of the reference sample was of $11 \times 10^{12} \mathrm{~m}^{-2}$, what is clearly of the level of the noise. This is consistent for this undeformed sample that contains no GNDs induced by creep.

For the deformed sample, the average GND density of $16.6 \times 10^{12} \mathrm{~m}^{-2}$ is higher than the noise. This was further quantified with the average Signal to Noise Ratio (SNR) expressed as $\left(\rho_{\mathrm{GNDs}} / \rho_{\mathrm{sp}}\right)^{2}$. 


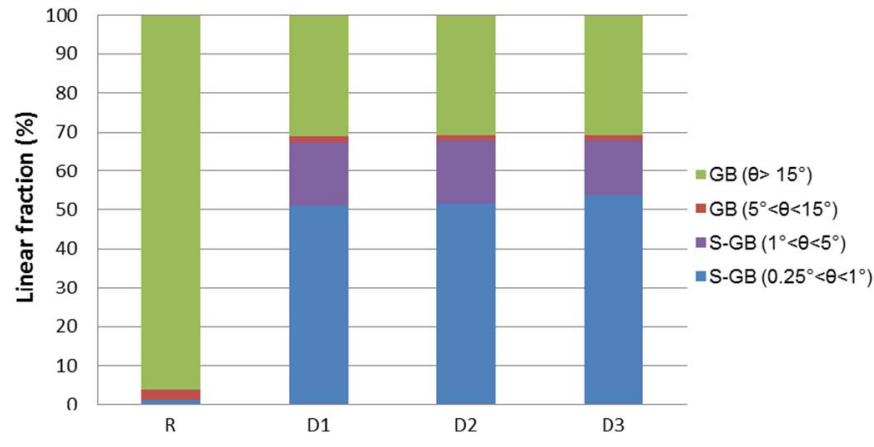

Fig. 9. Linear fractions of grain and sub-grain boundaries (GB and S-GB), measured by EBSD (filtered data) on the reference sample (R) and on three areas of the deformed sample (D1, D2, D3) (considering all disorientation lines with angles $>0.25^{\circ}$ ).

Table 1

Grain orientation spread evaluated from the EBSD map of the reference sample to estimate the disorientation range belonging to the noise.

\begin{tabular}{llllll}
\hline & \multicolumn{2}{l}{ Unfiltered data } & & \multicolumn{2}{l}{ Filtered data } \\
\cline { 2 - 3 } \cline { 5 - 6 } $\begin{array}{lllll}\mathrm{N}=200 \text { grains } \\
\text { (average diameter }>5 \mu \mathrm{m})\end{array}$ & A-GOS & M-GOS & & A-GOS & M-GOS \\
\hline Average disorientation & & & & \\
Standard deviation & $0.05^{\circ}$ & $0.17^{\circ}$ & & $0.016^{\circ}$ & $0.12^{\circ}$ \\
Average disorientation $+3 \sigma$ & $0.015^{\circ}$ & $0.05^{\circ}$ & & $0.01^{\circ}$ & $0.04^{\circ}$ \\
& $0.32^{\circ}$ & & $0.05^{\circ}$ & $0.25^{\circ}$ \\
\hline
\end{tabular}

One obtains a SNR of 2.1. This confirms that the GND density calculation is sensitive to the dislocation increase after $8 \%$ deformation by creep.

Table 2 also shows that filtering the data offers the opportunity to improve the SNR and makes the GND density calculation even more sensitive to low variations observed for different levels of creep deformation. Indeed, the average of spurious density $\rho_{\mathrm{sp}}$ on the filtered dataset was almost 20 times lower than the one obtained on the unfiltered dataset (see Table 2, $\rho_{\mathrm{sp}}=0.67 \times 10^{12} \mathrm{~m}^{-2}$ ). Of course, the average GND density value dropped also to $7.9 \times 10^{12} \mathrm{~m}^{-2}$. But the SNR increased to 140 . This drop by a factor of almost 20 for the spurious dislocation density between unfiltered and filtered data is surprisingly high (the corresponding average noise in Table 1 decreased only a half). Actually the local orientation averaging performed by the Kuwahara filter leads to neighboring pixels with zero-disorientations. They are translated in 'zero' dislocation density values (whereas a local spurious density of $\rho_{\mathrm{sp}} \approx 10^{12} \mathrm{~m}^{-2}$ is obtained from the local orientation noise). This explains the impressive drop in spurious dislocation density for the filtered data.

Our approach may also give new insight in the evaluation of the lower bound of the detectable dislocation density (i.e. level of spurious density). In the literature, this lower bound is often deduced from the estimated orientation noise by $[54,55]$ :

$\rho_{\text {sp }}=\frac{\theta_{n}}{|b| a}$

where $\theta_{n}$ is the noisy disorientation angle, $a$ the step size and $b$ the Burger's vector. How the disorientation noise has been evaluated is rarely explained. Some authors consider for $\theta_{n}$ a minimum angle of $1^{\circ}$ [55]. This value would result here in a dislocation density noise of $1.1 \times 10^{14} \mathrm{~m}^{-2}$. In a 3D experiment, Konijnenberg [54] assumes a much smaller $\theta_{n}$ of $0.2^{\circ}$ that would here give a dislocation density noise of $2.25 \times 10^{13} \mathrm{~m}^{-2}$. Often the authors compare spurious densities deduced from local disorientation noise with averaged dislocation densities.

For the present study, the average spurious density $\rho_{\mathrm{sp}}$ of $11.5 \times 10^{12} \mathrm{~m}^{-2}$ (Table 2, unfiltered data) gives $\theta_{n}=0.1^{\circ}$ according to Eq. (5). This value is clearly close to the average disorientation noise calculated in Table 1. The local disorientation noise of $0.25^{\circ}$ gives a local spurious density of $2.8 \times 10^{13} \mathrm{~m}^{-2}$. However this value is not an average value and cannot be compared to average GND density values (by the way, significantly below). Finally applying Eq. (5) to the filtered data $\left(\rho_{\mathrm{sp}}\right.$ of $0.67 \times 10^{12} \mathrm{~m}^{-2}$ ) gives an average disorientation noise of $0.006^{\circ}$ (6 times lower than the calculated value on the filtered data in Table 1). So, on filtered data, the $\theta_{n}$ value deduced from Eq. (5) definitely underestimates the average disorientation noise.

\section{Conclusion}

This contribution brings new insights to analyze and quantify the deformation substructure induced by dislocational creep, on the basis of advanced EBSD and A-ECCI experiments. It is applied to a porous $\mathrm{UO}_{2}$

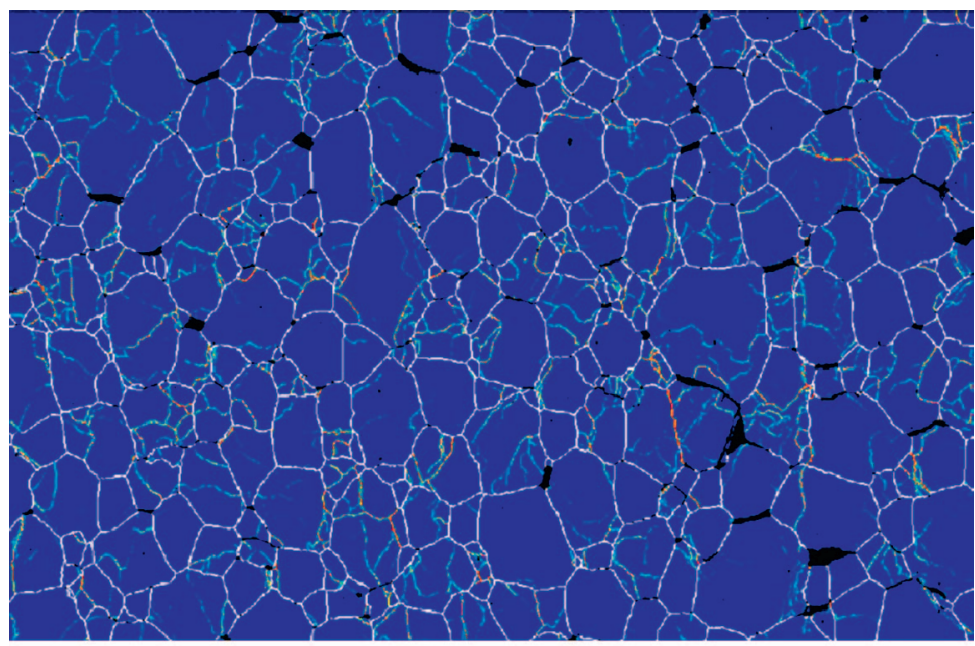

Fig. 10. Distribution of GND density (in terms of the entrywise1norm) for the deformed sample, calculated from the filtered EBSD data considering a cut-off angle of $5^{\circ}$ (boundaries with larger disorientations than $5^{\circ}$ are drawn in white - a linear color scale is used for the variation of dislocation densities).

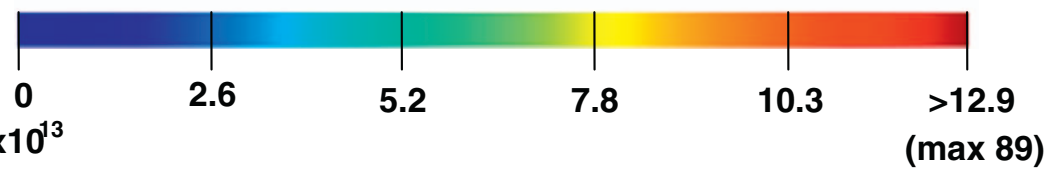


Table 2

Average dislocation densities $\left\langle\rho_{\mathrm{GND}}\right\rangle$ calculated from the EBSD maps of the reference and the deformed sample.

\begin{tabular}{lll}
\hline & Unfiltered dataset & Filtered dataset \\
\cline { 2 - 3 } & $\begin{array}{l}\text { Norm average } \\
\left(\times 10^{12} \mathrm{~m}^{-2}\right)\end{array}$ & $\begin{array}{l}\text { Norm average } \\
\left(\times 10^{12} \mathrm{~m}^{-2}\right)\end{array}$ \\
\hline Reference sample & 11 & 0.68 \\
Estimation of the spurious density & 11.5 & 0.67 \\
$\quad \rho_{\text {sp }}$ : (reference sample for & & \\
$\quad$ disorientations $\left.\left(0-0.25^{\circ}\right)\right)$ & 16.6 & 8 \\
Deformed map D1 & 16.1 & 8 \\
Deformed map D2 & 17 & 7.8 \\
Deformed map D3 & 16.6 & 7.9 \\
Deformed sample - average value & 2.1 & 140 \\
Signal to noise ratio: $\left(\rho_{\mathrm{GNDs}} / \rho_{\mathrm{sp}}\right)^{2}$ & & \\
\hline
\end{tabular}

pellet compression crept under $50 \mathrm{MPa}$ at $1500{ }^{\circ} \mathrm{C}$.

First, an optimized protocol for EBSD data acquisition and processing is proposed to efficiently quantify sub-boundaries in porous material. The application to $\mathrm{UO}_{2}$ pellets demonstrates that very low angle boundaries, disoriented down to $0.1^{\circ}$ can be reliably detected. This result corresponds to a real improvement to quantify sub-boundaries, in comparison to standard EBSD, less precise, or HR-EBSD, more time consuming. EBSD-based quantities calculated from large EBSD maps are further introduced to quantify the deformation substructure at a given creep state.

Moreover, evidence is made that A-ECCI is a powerful method to image the dislocations produced by creep and to analyze their arrangement in low angle boundaries. TEM quality images of dislocation networks are obtained on crept $\mathrm{UO}_{2}$, directly on the bulk material analyzed by EBSD.

So, both techniques, EBSD and A-ECCI complement each other to obtain a comprehensive analysis of the deformation substructure from the dislocation level to the polycrystalline one.

Finally, the $\mathrm{UO}_{2}$ deformation substructure after a given crept state ( $8 \%$ mean deformation, at $1500{ }^{\circ} \mathrm{C}$ under $50 \mathrm{MPa}$ ) is analyzed taking advantage of the scale transition offered by EBSD and A-ECCI. A dense network of sub-boundaries was induced by creep. After deformation, the sub-boundaries disoriented by $<5^{\circ}$ reached $67 \%$ of the total boundary length. Very low angle boundaries were also evidenced. They were formed by different arrangements of dislocations in low energy configurations. The prior $\mathrm{UO}_{2}$ grains, with an average size of $20 \mu \mathrm{m}$, were in average fragmented in sub-grains of about $5 \mu \mathrm{m}$. However, these sub-grains rarely formed a homogeneous network within the prior grains but had the tendency to concentrate at their boundaries. This network of sub-boundaries was further translated in GNDs. For the studied deformation state, the lower bound of the GND densities was estimated to $7.9 \times 10^{12} \mathrm{~m}^{-2}$ in average on the filtered EBSD data (for an average spurious density estimated to $0.67 \times 10^{12} \mathrm{~m}^{-2}$ ). This value was 10 times higher than that measured on an 'as sintered' reference sample.

Such analysis gives key results to quantify the deformation substructure and understand the material behavior. It can thus be very useful to compare the microstructure evolution, for different creep conditions. In case of sintered uranium dioxide fuel, it may help optimizing the microstructure to improve the viscoplastic behavior of the pellets during power transients.

\section{References}

[1] S. Aas, Mechanical interaction between fuel and cladding, Nucl. Eng. Des. 21 (1972) 237-253.

[2] B. Michel, J. Sercombe, C. Nonon, O. Fandeur, Modeling of pellet-cladding interaction, Compr. Nucl. Mater. 3 (2012) 677-712.

[3] F. Dherbey, F. Louchet, A. Mocellin, S. Leclercq, Elevated temperature creep of polycrystalline uranium dioxide: from microscopic mechanisms to macroscopic behaviour, Acta Mater, 50 (2002) 1495-1505.

[4] A. Alamo, J.M. Lefebvre, J. Soullard, Déformation plastique du bioxyde d'uranium: observation des sous-structures de dislocations, J. Nucl. Mater. 75 (1978) 145-153.

[5] D.R. Clarke, Observation of crystal defects using scanning electron microscope, Philos. Mag. 24 (1971) 973-979.

[6] R.M. Stern, T. Ichinokawa, S. Takashima, H. Hashimoto, S. Kimoto, Dislocation images in high-resolution scanning electron-microscope, Philos. Mag. 26 (1972) 1495-1499.

[7] D.C. Joy, Direct defect imaging in the high resolution SEM, Mater. Res. Soc. Symp. Proc. 183 (1990) 199-210.

[8] B.A. Simkin, M.A. Crimp, An experimentally convenient configuration for electron channeling contrast imaging, Ultramicroscopy 77 (1999) 65-75.

[9] B.C. Ng, B.A. Simkin, M.A. Crimp, Application of the electron channeling contrast imaging technique to the study of dislocations associated with cracks in bulk specimens, Ultramicroscopy 75 (1998) 137-145.

[10] M. Pitaval, P. Morin, J. Baudry, G. Fontaine, Energy filtering of backscattered electrons in scanning electron microscopy, Microsc. Spect. Elec. 2 (1977) 185-196.

[11] P. Morin, M. Pitaval, D. Besnard, G. Fontaine, Electron-channeling imaging in scanning electron-microscopy, Philos. Mag. A 40 (1979) 511-524.

[12] D.C. Joy, D.E. Newbury, D.L. Davidson, Electron channeling patterns in the scanning electron microscope, J. Appl. Phys. 53 (1982) 81-122.

[13] J.T. Czernuszka, N.J. Long, P.B. Hirsch, Electron channelling contrast imaging of dislocations, Proceedings of the 12th International Congress for Electron Microscopy, 410 San Francisco Press, 1990.

[14] B.A. Simkin, B.C. Ng, T.R. Bieler, M.A. Crimp, D.E. Mason, Orientation determination and defect analysis in the near-cubic intermetallic $\gamma$-TiAl using SACP, ECCI, and EBSD, Intermetallics 11 (2003) 215-223.

[15] H. Kriaa, A. Guitton, N. Maloufi, Fundamental and experimental aspects of diffraction for characterizing dislocations by electron channeling contrast imaging in scanning electron microscope, Sci Rep 7 (2017) 9742.

[16] Y.N. Picard, M. Liu, J. Lammatao, R. Kamaladasa, M. De Graef, Theory of dynamical electron channeling contrast images of near-surface crystal defects, Ultramicroscopy 146 (2014) 71.

[17] A.J. Wilkinson, P.B. Hirsch, The effects of surface stress relaxation on electron channelling contrast images of dislocations, Philos. Mag. E 72 (1995) 81-103.

[18] N. Ferralis, J. Kawasaki, R. Maboudian, C. Carraro, Evolution in surface morphology of epitaxial graphene layers on $\mathrm{SiC}$ induced by controlled structural strain, Appl. Phys. Lett. 93 (2008) 191916.

[19] A. Barnoush, Correlation between dislocation density and nanomechanical response during nanoindentation, Acta Mater. 60 (2012) 1268-1277.

[20] J. Ahmed, S.G. Roberts, A.J. Wilkinson, Characterizing dislocation structure evolution during cyclic deformation using electron channelling contrast imaging, Philos. Mag. 86 (2006) 4965-4981.

[21] M. Marx, W. Schaef, M.T. Welsch, The microstructure as crack initiation point and barrier against fatigue damaging, Int. J. Fatigue 41 (2012) 57-63.

[22] J. Ahmed, A.J. Wilkinson, S.G. Roberts, Study of dislocation structures near fatigue cracks using electron channelling contrast imaging technique (ECCI), J. Microsc. 195 (1999) 197-203.

[23] Y.N. Picard, R. Kamaladasa, M. De Graef, N.T. Nuhfer, W.J. Mershon, T. Owens, L. Sedlacek, F. Lopour, Future prospects for defect and strain analysis in the SEM via electron channeling, Microsc. Today 20 (2012) 12-16.

[24] G. Naresh-Kumar, B. Hourahine, P.R. Edwards, A.P. Day, A. Winkelmann, A.J. Wilkinson, P.J. Parbrook, G. England, C. Trager-Cowan, Rapid nondestructive analysis of threading dislocations in Wurtzite materials using the scanning electron microscope, Phys. Rev. Lett. 108 (2012) 135503.

[25] S. Zaefferer, N.N. Elhami, Theory and application of electron channelling contrast imaging under controlled diffraction conditions, Acta Mater. 75 (2014) 20-50.

[26] J. Guyon, H. Mansour, N. Gey, M.A. Crimp, S. Chalal, N. Maloufi, Sub-micron resolution selected area electron channeling patterns, Ultramicroscopy 149 (2015) 34-44.

[27] H. Mansour, J. Guyon, M.A. Crimp, N. Gey, B. Beausir, N. Maloufi, Accurate electron contrast analysis of dislocations in fine grained bulk materials, Scr. Mater. 84 85 (2014) 11-14.

[28] X. Iltis, M. Ben Saada, H. Mansour, N. Gey, A. Hazotte, N. Maloufi, A new characterization approach for studying relationships between microstructure and creep damage mechanisms of uranium dioxide, J. Nucl. Mater. 474 (2016) 1-7.

[29] D.J. Dingley, Progressive steps in the development of electron backscatter diffraction and orientation imaging microscopy, J. Microsc. (Oxford) 213 (2004) 214-224.

[30] F.J. Humphreys, Review - Grain and subgrain characterisation by electron backscatter diffraction, J. Mater. Sci. 36 (2001) 3833-3854.

[31] M. Ben Haj Slama, N. Gey, L. Germain, J.C. Hell, K. Zhu, S. Allain, Fast granularization of lath - like bainite in $\mathrm{FeNiC}$ alloys during isothermal holding at Ms $+20{ }^{\circ} \mathrm{C}$, Metall. Mater. Trans. A 47 (2016) 15-18.

[32] W. Pantleon, Resolving the geometrically necessary dislocation content by conventional electron backscattering diffraction, Scr. Mater. 58 (2008) 994-997.

[33] B. Beausir, C. Fressengeas, Disclination densities from EBSD orientation mapping, Int. J. Solids Struct. 50 (2013) 137-146.

[34] A. Ndiaye, Microstructures d' $\mathrm{UO}_{2}$ à propriétés pilotées : compréhension des mécanismes d'élaboration et du comportement mécanique en température, $\mathrm{PhD}$ Thesis University of Grenoble, France, 2012.

[35] K. Thomsen, N.H. Schmidt, Improving the Accuracy of Orientation Measurements Using EBSD, Microsc. Microanal. 19 (Suppl 2) (2013).

[36] B. Beausir, J.-J. Fundenberger, Université de Lorraine - Metz, ATOM - analysis tools for orientation maps, http://atom-software.eu/, (2015).

[37] M. Kuwahara, K. Hachimura, S. Eiho, M. Kinoshita, Processing of RI-angiocardiographic images, in: K. Preston, Jr.M. Onoe (Eds.), Digital Processing of Biomedical 
Images, Plenum, New York, 1976, pp. 187-202.

[38] F.J. Humphreys, P.S. Bate, P.J. Hurley, Orientation averaging of electron back scattered diffraction data, J. Microsc. 201 (2001) 50-58.

[39] H. Mansour, M.A. Crimp, N. Gey, N. Maloufi, Accurate electron channeling contrast analysis of a low angle sub-grain boundary, Scr. Mater. 109 (2015) 76-79.

[40] A. Winkelmann, C. Trager-Cowan, F. Sweeney, A.P. Day, P. Parbrook, Many-beam dynamical simulation of electron backscatter diffraction patterns, Ultramicroscopy 107 (2007) 414-421.

[41] C. Maurice, R. Fortunier, A 3D Hough transform for indexing EBSD and Kossel patterns, J. Microsc. 230 (2008) 520-529.

[42] A.J. Wilkinson, G. Meaden, D.J. Dingley, High-resolution elastic strain measurement from electron backscatter diffraction patterns: new levels of sensitivity, Ultramicroscopy 106 (2006) 307-313.

[43] C. Maurice, R. Quey, R. Fortunier, J.H. Driver, High angular resolution EBSD and its materials applications, in: Dmitri A. Molodov (Ed.), Microstructural Design of Advanced Engineering Materials, 2013, pp. 339-365.

[44] T.B. Britton, C. Maurice, R. Fortunier, J.H. Driver, A.P. Day, G. Meaden, D.J. Dingley, K. Mingard, A.J. Wilkinson, Factors affecting the accuracy of high resolution electron backscatter diffraction when using simulated patterns, Ultramicroscopy 110 (2010) 1443-1453.

[45] L. Priester, Grain boundaries: from theory to engineering, Mechanical Stress Order of Grain Boundaries, 172 Springer, 2012, pp. 29-47.

[46] J. Friedel, Dislocations, Pergamon Press, Oxford_New York, 1964.

[47] E.J. Rapperport, A.M. Huntress, Deformation modes of single crystal uranium dioxide from $700{ }^{\circ} \mathrm{C}$ to $1900{ }^{\circ} \mathrm{C}$, Nuclear Metals (USA) Report NMI-1242, 1960, pp. $1-29$.

[48] P.T. Sawbridge, E.C. Sykes, Electrostatic charges on dislocations in uranium dioxide, J. Nucl. Mater. 35 (1970) 122-125.

[49] J. Soulacroix, B. Michel, J.M. Gatt, R. Kubler, L. Barrallier, Micromechanical
Behavior of $\mathrm{UO}_{2}$ : Crystalline Anisotropy and Associated Internal Stresses in Polycrystals, 21ème Congrès Français de Mécanique, Bordeaux, 2013.

[50] L. Germain, D. Kratsch, M. Salib, N. Gey, Identification of sub-grains and low angle boundaries beyond the angular resolution of EBSD maps, Mater. Charact. 98 (2014) 66-72.

[51] Disclosed anonymously, grain boundary completion, Res. Discl. (2013), www. researchdisclosure.com.

[52] W. Pantleon, Resolving the geometrically necessary dislocation content by conventional electron backscattering diffraction, Scr. Mater. 58 (2008) 994-997.

[53] J. Nye, Some geometrical relations in dislocated crystals, Acta Metall. 1 (1953) 153-162.

[54] P.J. Konijnenberg, S. Zaefferer, D. Raabe, Assessment of geometrically necessary dislocation levels derived by 3D EBSD, Acta Mater. 99 (2015) 402-414.

[55] W. He, W. Ma, W. Pantleon, Microstructure of individual grains in cold-rolled aluminium from orientation inhomogeneities resolved by electron backscattering diffraction, Mater. Sci. Eng. A 494 (2008) 21-27.

[56] D.P. Field, P.B. Trivedi, S.I. Wright, M. Kumar, Analysis of local orientation gradients in deformed single crystals, Ultramicroscopy 103 (2005) 33-39.

[57] J. Jiang, T.B. Britton, A.J. Wilkinson, Measurement of geometrically necessary dislocation density with high resolution electron backscatter diffraction: effects of detector binning and step size, Ultramicroscopy 125 (2013) 1-9.

[58] J.W. Kysar, Y. Saito, M.S. Oztop, D. Lee, W.T. Huh, Experimental lower bounds on geometrically necessary dislocation density, Int. J. Plast. 26 (2010) 1097-1123.

[59] T.J. Ruggles, T.M. Rampton, A. Khosravani, D.T. Fullwood, The effect of length scale on the determination of geometrically necessary dislocations via EBSD continuum dislocation microscopy, Ultramicroscopy 164 (2016) 1-10.

[60] S.I. Wright, D.P. Field, M.M. Nowell, Post processing effects on GND calculations from EBSD-based orientation measurements, Mater. Sci. Eng. 89 (2015) 012049. 